\title{
Thermal characteristics of the Airy wall jet for constant surface heat
} flux

\section{Journal Article}

Author(s):

Magyari, E.; Weidman, P.D.

Publication date:

2006-07

Permanent link:

https://doi.org/10.3929/ethz-b-000036917

Rights / license:

In Copyright - Non-Commercial Use Permitted

Originally published in:

Heat and Mass Transfer 42(9), https://doi.org/10.1007/s00231-005-0048-8 


\section{ORIGINAL}

\section{E. Magyari • P. D. Weidman}

\section{Thermal characteristics of the Airy wall jet for constant surface heat flux}

Received: 14 January 2005 / Accepted: 26 October 2005/Published online: 1 December 2005

(C) Springer-Verlag 2005

\begin{abstract}
The Airy jet is a wall-bounded flow belonging to the similarity class of the well known free jet but, in contrast to the latter, its far field behavior is an algebraically decaying rotational flow. The present paper investigates the thermal characteristics of the Airy jet over a wall with prescribed constant heat flux. The scaling behavior found for small and large values of the Prandtl number is compared to those obtained earlier for (a) the case of a wall with prescribed constant temperature and for (b) the case of a preheated Airy jet adjacent to an insulated wall.
\end{abstract}

\section{List of symbols}

$A i, B i \quad$ Airy functions

$c_{p} \quad$ specific heat at constant pressure

$f(\eta) \quad$ similar stream function, Eq. 3

$G$ normalized temperature variable, Eq. 15

$k$ thermal conductivity

$L \quad$ reference length

$\mathrm{Nu} \quad$ Nusselt number

$\mathrm{Pr} \quad$ Prandtl number

$q \quad$ heat flux

$Q \quad$ convected heat flux, Eq. 13

$T$ temperature

$T_{*} \quad$ reference temperature

$u \quad$ streamwise velocity component

$x, y \quad$ dimensionless Cartesian coordinates

$z \quad$ argument of the Airy functions, Eq. 3

E. Magyari $(\bowtie)$

Chair of Physics of Buildings, Institute of Building Technology,

Swiss Federal Institute of Technology (ETH) Zürich,

8093 Zürich, Switzerland

E-mail: magyari@hbt.arch.ethz.ch

Tel.: + 41-1-6332867

Fax: + 41-1-6331041

P. D. Weidman

Department of Mechanical Engineering, University of Colorado, Boulder, CO 80309-0427, USA

\author{
Greek symbols \\ $\gamma \quad$ power-law exponent, Eq. la \\ $\Gamma \quad$ Gamma function \\ $\eta \quad$ independent similarity variable, Eq. $1 \mathrm{~b}$ \\ $\vartheta(\eta) \quad$ similarity temperature variable, Eq. 1a \\ $\theta$ modified similarity temperature variable, \\ Eqs. 18 and 19 \\ v kinematic viscosity \\ $\rho \quad$ density
}

Subscripts and superscripts
$\begin{array}{ll}\text { ad } & \text { adiabatic } \\ \text { w } & \text { wall conditions } \\ \infty & \text { far field condition } \\ , & \text { derivative with respect to } \eta \text { or } z\end{array}$

\section{Introduction}

The algebraically decaying Airy wall jet has first been considered by Weidman et al. [1] on impermeable and more recently by Magyari et al. [2] on permeable walls. Some of its thermal characteristics for prescribed constant wall temperature and an insulated wall were reported by Magyari et al. [3]. The thermal characteristics of the preheated Airy jet were further investigated and compared in detail with those of the classical (exponentially decaying) Tetervim [4], Akatnov [5] and Glauert [6] wall jet by Magyari and Weidman [7].

The present paper investigates the thermal characteristics of the Airy jet over a wall with prescribed constant heat flux and compares the scaling behavior found for small and large values of the Prandtl number to those obtained for (a) the case of a wall with prescribed constant temperature [3] and for (b) the case of a preheated Airy jet adjacent to an insulated wall [7]. 


\section{Similarity formulation and the Airy jet solution [3]}

The Airy wall jet of incoming heat flux $Q$ per unit span issuing through a narrow slot is considered. The $z$-axis lies along the slot, and $x$ and $y$ are the dimensionless streamwise and wall normal Cartesian coordinates, respectively. For incompressible flow in the boundary layer approximation, neglecting buoyancy and viscous self-heating effects, the governing equations for the corresponding similar temperature field are of the form [3]:

$T(x, y)=T_{\infty}+T_{*} x^{\gamma} \vartheta(\eta), \quad \eta=x^{-2 / 3} y$

$\frac{3}{P r} \vartheta^{\prime \prime}+f \vartheta^{\prime}-3 \gamma f^{\prime} \vartheta=0$

Here $f$ is the similar stream function of the Airy wall jet [3],

$f(\eta)=(24)^{1 / 3} \frac{\sqrt{3} A i^{\prime}(z)+B i^{\prime}(z)}{\sqrt{3} A i(z)+B i(z)}, \quad z=3^{-2 / 3} \eta$

corresponding to the asymptotic condition $f^{\prime}(\eta)=\eta^{-1 / 2}$ as $\eta \rightarrow \infty$ (i.e., to the value 1 of the parameter $\beta$ of [3]).

\section{Temperature solutions}

The coupling of the thermal boundary-value problems to the flow solution (3) for the three cases of interest are described below.

\subsection{Case I: prescribed constant wall heat flux}

Having in mind that

$q_{\mathrm{w}}(x)=-\left(k T_{*} / L\right) x^{\gamma-2 / 3} \vartheta^{\prime}(0)$

the temperature exponent $\gamma=2 / 3$ is required for $q_{\mathrm{w}}=$ constant. Thus, the corresponding thermal boundary value problem is

$\frac{3}{P r} \vartheta^{\prime \prime}+f \vartheta^{\prime}-2 f^{\prime} \vartheta=0$

$\vartheta^{\prime}(0)=-1, \quad \vartheta(\infty)=0$

The quantity of physical interest is the temperature field (1a), where the reference temperature $T_{*}$ is specified by the prescribed value of the wall heat flux, $q_{\mathrm{w}}=k T_{*} / L$. In this way Eq. 1a gives

$T=T_{\infty}+\frac{q_{\mathrm{w}} L}{k} x^{2 / 3} \vartheta(\eta)$

and the wall temperature distribution is

$T_{\mathrm{w}}=T_{\infty}+\frac{q_{\mathrm{w}} L}{k} x^{2 / 3} \vartheta(0)$

The dimensionless wall temperature $\vartheta(0)$ depends on the Prandtl number. This functional dependence is obtained from the solution of the boundary value problem (5) (see Section 4).

3.2 Case II: prescribed constant wall temperature [3]

According to Eq. 1a, the temperature exponent $\gamma=0$ is required for constant wall temperature. Thus, the corresponding thermal boundary value problem is

$\frac{3}{\operatorname{Pr}} \vartheta^{\prime \prime}+f \vartheta^{\prime}=0$

$\vartheta(0)=+1, \quad \vartheta(\infty)=0$

The temperature field is given by Eq. 1a, where the reference temperature $T *$ is specified by the prescribed value $T_{\mathrm{w}}$ of the wall temperature. In this way, $T_{*}=T_{\mathrm{w}}-T_{\infty}$ and

$T=T_{\infty}+\left(T_{\mathrm{w}}-T_{\infty}\right) \vartheta(\eta)$

The quantity of engineering interest is the wall heat flux distribution, which is now given by

$q_{\mathrm{w}}(x)=-\frac{k\left(T_{\mathrm{w}}-T_{\infty}\right)}{L} x^{-2 / 3} \vartheta^{\prime}(0)$

As shown in [3] the dimensionless wall temperature $\vartheta(\eta)$ and the dimensionless wall temperature gradient $\vartheta^{\prime}(0)$ can be obtained by quadratures, viz.

$\vartheta(\eta)=1-\frac{\int_{0}^{z}[\sqrt{3} A i(z)+B i(z)]^{-2 P r} \mathrm{~d} z}{\int_{0}^{\infty}[\sqrt{3} A i(z)+B i(z)]^{-2 P r} \mathrm{~d} x}$
$\vartheta^{\prime}(0)=-3^{-2 / 3} \frac{[\sqrt{3} A i(0)+B i(0)]^{-2 P r}}{\int_{0}^{\infty}[\sqrt{3} A i(z)+B i(z)]^{-2 P r} \mathrm{~d} z}$

where $\sqrt{3} A i(0)+B i(0)=2 \times 3^{-1 / 6} / \Gamma(2 / 3)[8]$.

3.3 Case III: insulated wall [7]

The requirement that the incoming heat flux per unit span

$Q=\rho c_{p} \int_{0}^{\infty} u \cdot\left(T-T_{\infty}\right) \mathrm{d} y$

is an integral invariant, selects for the temperature exponent the value $\gamma=-1 / 3$. Thus the corresponding thermal boundary value problem is

$\left(3 \vartheta^{\prime}+\operatorname{Prf} \vartheta\right)^{\prime}=0$

$\vartheta^{\prime}(0)=0, \quad \vartheta(\infty)=0$

As shown in [7], the dimensionless wall temperature $\vartheta$ $(\eta)$ is given in this case by

$\vartheta(\eta)=\vartheta(0)\left[\frac{\sqrt{3} A i(0)+B i(9)}{\sqrt{3} A i(z)+B i(z)}\right]^{2 P r} \equiv \vartheta(0) G(\eta)$

where $\vartheta(0)$ is still undetermined. Here the temperature field is given by 
$T=T_{\infty}+T_{*} x^{-1 / 3} \vartheta(0) G(\eta)$

The product $T_{*} g(0)$ of the unknown constants can uniquely be determined by specifying the incoming heat flux $Q_{0}$ and substituting Eq. 16 and $u=(v / L) x^{-1 / 3} f^{\prime}(\eta)$ into Eq. 13. The result reads

$T_{*} g(0)=\frac{Q_{0}}{v \rho c_{p}} \theta_{\text {ad }}(P r)$

where

$\theta_{\mathrm{ad}}(P r)=\left(\int_{0}^{\infty} f^{\prime} G \mathrm{~d} \eta\right)^{-1}$

is the dimensionless adiabatic temperature of the wall. Accordingly, the temperature distribution along the insulated wall below the preheated jet is

$T_{\mathrm{w}}(x)=T_{\infty}+\frac{Q_{0}}{v \rho c_{p}} x^{-1 / 3} \theta_{\mathrm{ad}}(P r)$

\section{Dependence on the PrandtI number}

In this section the asymptotic variations of relevant heat transfer properties, for small and large Prandtl numbers, are obtained from numerical solutions of the thermal boundary-value problem for the three cases of interest.

\subsection{Case I: prescribed constant wall heat flux}

The quantity of interest here is the dimensionless wall temperature $\vartheta(0)$ as a function of the Prandtl number, obtained from the numerical solution of the boundaryvalue problem (5). The result is displayed in Fig. 1 where $1 / \vartheta(0)$ has been plotted as a function of Pr. Numerical computations furnish the following scaling laws for asymptotically small and large values of the Prandtl number:

$\frac{1}{\vartheta(0)} \rightarrow\left\{\begin{array}{lll}1.56018 \cdot \operatorname{Pr}^{2 / 3} & \text { as } & \operatorname{Pr} \rightarrow 0 \\ 0.60183 \cdot \operatorname{Pr}^{1 / 3} & \text { as } & \operatorname{Pr} \rightarrow \infty\end{array}\right.$

The Nusselt number $N u=\left(q_{\mathrm{w}} L / k\right) /\left(T_{\mathrm{w}}-T_{\infty}\right)$ for this case is

$N u_{\mathrm{I}}=\frac{x^{-2 / 3}}{\vartheta(0)}$

It is worth noticing that the asymptotic formula (20) for large $\mathrm{Pr}$ provides reasonably accurate results down to $O$ (1) values of $P r$; for $P r=1$, e.g., the approximate value $1 / \vartheta(0)=0.60183$ exceeds the (numerically) exact value $1 /$ $\vartheta(0)=0.589282$ only by $2 \%$.

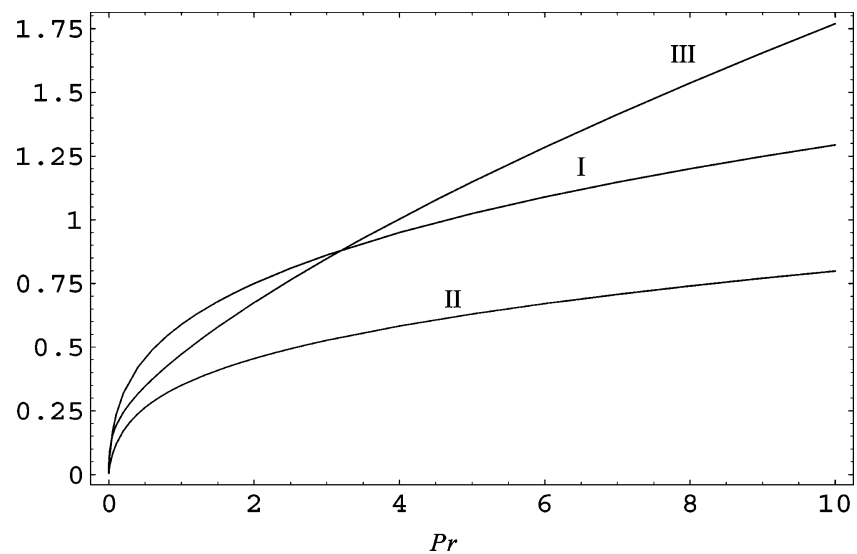

Fig. 1 Dependence of the thermal characteristics of the Airy wall jet on the Prandtl number: Curve I Plot of $1 / \vartheta(0)$ for constant wall heat flux; see Eqs. 7, 20, and 21; Curve II Plot of $-\vartheta^{\prime}(0)$ for constant wall temperature; see Eqs. 10, 12, 22, and 23; Curve III Plot of $\theta_{\text {ad }}$ for the preheated jet over an insulated wall; see Eqs. 18, 19 , and 24

\subsection{Case II: prescribed constant wall temperature}

The plot of the dimensionless wall temperature gradient (12) as a function of $\mathrm{Pr}$ is also shown in Fig. 1. These numerical solutions of boundary-value problem (8) yield the following large and small Prandtl number asymptotic behaviors

$-\vartheta^{\prime}(0) \rightarrow\left\{\begin{array}{lll}0.64513 \cdot \operatorname{Pr}^{2 / 3} & \text { as } & \operatorname{Pr} \rightarrow 0 \\ 0.37328 \cdot \operatorname{Pr}^{1 / 3} & \text { as } & \operatorname{Pr} \rightarrow \infty\end{array}\right.$

and the Nusselt number for this constant wall temperature problem is

$N u_{\text {II }}=x^{-2 / 3}\left[-\vartheta^{\prime}(0)\right]$

Again, for large values of $\operatorname{Pr}$ the asymptotic formula (22) furnishes quite accurate estimates down to $O(1)$ values of $P r$; for $P r=3$, e.g., the approximate value $\vartheta^{\prime}(0)=0.538363$ exceeds the (numerically) exact value $\vartheta^{\prime}(0)=0.526761$ only by $2.2 \%$.

\subsection{Case III: insulated wall}

For comparison, we have included in Fig. 1 the Prandtl number variation of dimensionless adiabatic wall temperature (18) reported in [7]. As shown in [7], the asymptotic behaviors of $\theta_{\mathrm{w}}(P r)$ for small and large $P r$ are given by:

$\theta_{\mathrm{ad}}(\operatorname{Pr}) \rightarrow\left\{\begin{array}{llc}0.42731 \cdot \operatorname{Pr}^{1 / 3} & \text { as } & \operatorname{Pr} \rightarrow 0 \\ 0.36924 \cdot \operatorname{Pr}^{2 / 3} & \text { as } & \operatorname{Pr} \rightarrow \infty\end{array}\right.$

\section{Summary and conclusions}

The thermal characteristics of Airy wall jets for constant wall heat flux $q_{\mathrm{w}}$, for constant wall temperature $T_{\mathrm{w}}$, and 
Table 1 Overview of the Prandtl number dependence of the thermal characteristics of the Airy wall jet

\begin{tabular}{lll}
\hline Thermal characteristics & $\operatorname{Pr} \rightarrow 0$ & $\operatorname{Pr} \rightarrow \infty$ \\
\hline$q_{\mathrm{w}}=$ const. $N u_{\mathrm{I}}=x^{-2 / 3} \vartheta(0)$ & $1 / \vartheta(0)=1.56018 \cdot \operatorname{Pr}^{2 / 3}$ & $1 / \vartheta(0)=0.60183 \cdot \operatorname{Pr}^{1 / 3}$ \\
$T_{\mathrm{w}}=$ const. $N u_{\mathrm{II}}=x^{-2 / 3}\left[-\vartheta^{\prime}(0)\right]$ & $-\vartheta^{\prime}(0)=0.64513 \cdot \operatorname{Pr}^{2 / 3}$ & $-\vartheta^{\prime}(0)=0.37328 \cdot \operatorname{Pr}^{1 / 3}$ \\
Adiabatic wall $\left(q_{\mathrm{w}}=0\right) \frac{T_{\mathrm{w}}(x)-T_{\infty}}{\left(Q_{0} / \nu \rho c_{p}\right)}=x^{-1 / 3} \theta_{\mathrm{ad}}(P r)$ & $\theta_{\mathrm{ad}}=0.42731 \cdot \operatorname{Pr}^{1 / 3}$ & $\theta_{\mathrm{ad}}=0.36924 \cdot \operatorname{Pr}^{2 / 3}$ \\
\hline
\end{tabular}

for a preheated jet flowing over an insulated wall are summarized in Table 1 in which their streamwise dependence and asymptotic Prandtl number scalings are compared. The explicit numerical variations of the relevant dimensionless quantities $1 / \vartheta(0),-\vartheta^{\prime}(0)$, and $\theta_{\mathrm{ad}}$ in the range $0<P r \leq 10$ are plotted in Fig. 1 .

Table 1 shows that the Nusselt numbers $N u_{\mathrm{I}}$ and $N u_{\mathrm{II}}$ scale with $\operatorname{Pr}$ and $x$ in the same manner. Thus, their ratio goes to constant values at all downstream stations $x$ for both small and large values of the Prandtl number

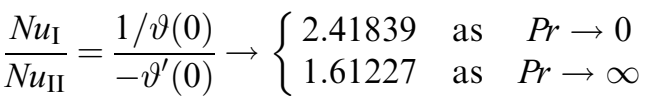

A notable feature of the thermally active Airy jet is that the scaling behavior of the wall temperature distribution both with respect to $P r$ and $x$ is quite different for a constant wall heat flux $q_{\mathrm{w}}$ compared to the preheated jet flowing over an insulated wall where $q_{\mathrm{w}}=0$; see Eqs. 7 and 19 and the first and third rows of Table 1.

\section{References}

1. Weidman PD, Kubitschek DG, Brown SN (1997) Boundary layer similarity flow driven by power-law shear. Acta Mech 120:199-215

2. Magyari E, Keller B, Pop I (2003) Boundary layer similarity flow driven by a power-law shear over a permeable plane surface. Acta Mech 163:139-146

3. Magyari E, Keller B, Pop I (2004) Heat transfer characteristics of a boundary layer flow driven by a power-law shear over a semi-infinite flat plate. Int J Heat Mass Transfer 47:31-34

4. Tetervin N (1948) Laminar flow of a slightly viscous incompressible fluid that issues from a slit and passes over a flat plate. NACA TN 1644, Washington, DC, p 40

5. Akatnov NI (1953) Development of 2D laminar jet along a solid surface. Leningrad Politek Inst Trudy 5:24-31

6. Glauert MB (1956) The wall jet. J Fluid Mech 1:625-643

7. Magyari E, Weidman PD (2005) The preheated Airy wall jet. Heat Mass Transfer 41:1014-1020

8. Abramowitz M, Stegun I (1972) Handbook of mathematical functions. U.S. Government Printing Office, Washington, DC 